\title{
Quality of Extracranial Carotid Evaluation with 256-Section CT
}

\author{
J.M. Johnson, M.S. Reed, H.N. Burbank, and C.G. Filippi
}

\begin{abstract}
BACKGROUND AND PURPOSE: To date, no systematic evaluation of image quality has been performed on the 256-section multidetector CT scanner for extracranial carotid evaluations. We evaluated image quality, patient dose, and examination time and compared these parameters with a 64-section multidetector CT.
\end{abstract}

MATERIALS AND METHODS: We reviewed extracranial CTA scans obtained on a 256-detector CT scanner in 50 consecutive patients. Image quality was analyzed for artifacts and vessel contrast opacification from the aortic arch to the skull base, dose to patient, and scan time. Results were compared with a control group of 50 patients imaged on a 64-section CT scanner. A Fisher exact test was used to analyze both groups with respect to vessel contrast opacification and presence of artifacts, and a Student $t$ test was used to assess differences in patient dose between the 2 groups.

RESULTS: Quantitative and qualitative evaluations revealed $>95 \%$ acceptable vessel opacification at all levels measured on the 256 section scanner. Scan time was improved (4 seconds on 256-channel). There were fewer artifacts related to metallic streak on the 256-channel CTA study, and DLP was lower on the 256-channel CTA (113.9 versus $159.8 \mathrm{mGy}$ ).

CONCLUSIONS: The 256-channel CTA imaging protocol for carotid arteries yielded similar vessel contrast opacification compared with the 64-channel CTA but with fewer metallic artifacts, a modest decrease in scan time, similar image quality, and a statistically significant reduction in radiation dose of $10 \%$.

ABBREVIATIONS: CCA = common carotid artery; $\mathrm{MDCT}=$ multidetector $\mathrm{CT} ; \mathrm{IJV}=$ internal jugular vein; $\mathrm{DLP}=$ dose-length product; $\mathrm{MIP}=$ maximal intensity projection

$\mathbf{S}$ troke is currently the second leading cause of death in the world, causing 9\%, with heart disease and cancer, respectively, ranking first and third. ${ }^{1}$ Approximately 795,000 people have a new or recurrent stroke each year in the United States. ${ }^{2}$ During the past decade, new management guidelines and treatments for acute brain infarct and extracranial arterial stenoses have been developed. This has led to an increasing demand for accurate and reliable imaging techniques to rapidly identify appropriate patients for these treatments. ${ }^{3-6}$

Although carotid sonography is considered the optimal noninvasive technique for screening of the proximal carotid arteries

Received July 24, 2012; accepted after revision October 23

From the Section of Neuroradiology (J.M.J., M.S.R., H.N.B., C.G.F.), Department of Radiology, Fletcher Allen Health Care, Burlington, Vermont; and Department of Radiology (J.M.J.), Massachusetts General Hospital and Harvard Medical School, Boston, Massachusetts.

Please address correspondence to Jason M. Johnson, MD, Massachusetts General Hospital, Department of Radiology, FND216, Boston, MA 02114-9657; e-mail: Johnson57@partners.org

http://dx.doi.org/10.3174/ajnr.A3433 and bifurcations, ${ }^{7}$ there are many situations in which sonography is suboptimal. Well-recognized limitations of carotid sonography include the inability to insonate the CCA origin or the ICA at the skull base as well as flow limitations as seen, for example, during the imaging of ICA tandem lesions, along with technical challenges related to insonating in the region of heavily calcified plaque. ${ }^{8}$ In addition, ultrasonographers with training in vascular techniques may not be readily available for urgent cerebrovascular examinations, and, even if available, the longer length of the examination compared with CT may make the test unsuitable for evaluation of the patient for emergent revascularization.

The major advantages of CTA include speed of acquisition, the ability to generate accurate anatomic images, and relatively low risk compared with conventional angiography. In addition, CTA provides anatomic information that is not as artifact-prone as flow-dependent MRA or Doppler ultrasound. ${ }^{9}$ MDCT has rapidly evolved from 4-detector systems in 1998 to 128-, 256-, and 320-detector systems. ${ }^{10}$ These newer-generation CT scanners offer advantages in scanning times, detector coverage, and resolution and decreased radiation dose. ${ }^{11}$ As temporal resolution and 
scanner speed improve, achieving optimal timing to ensure appropriate arterial opacification from the contrast bolus becomes increasingly difficult. This issue is especially relevant with polydetector (>64-section) scanners, in which the rapid CT acquisition can outpace the flow of contrast-opacified blood. Polydetector CT scanners have been available since $2008 .{ }^{10}$ At the study institution, a 256-section scanner came into routine clinical use in 2009 , when it became the primary scanner for all emergency cerebrovascular evaluations. Given the increased prevalence of polydetector scanners in clinical practice, the purpose of this article was to analyze both quantitatively and qualitatively CTA of the extracranial carotid arteries performed with 256-section MDCT compared with CTA by use of 64-section MDCT, with particular attention given to potential artifacts, scan time, dose to patient, and quantitative vessel opacification from the aortic arch to the skull base. In addition, we describe our recommended carotid CTA imaging protocol for 256-section MDCT scanners and discuss the limitations and artifacts unique to polydetector scanners.

\section{MATERIALS AND METHODS \\ The 256-Detector Protocol}

The 256-section CTA protocol used at our institution for evaluation of the extracranial carotid arteries is often performed as part of a comprehensive cerebrovascular examination consisting of 3 series obtained in the following order: 1) unenhanced head CT, 2) perfusion CT, and 3) CTA from the aortic arch through the cerebral vertex. After the unenhanced head CT, perfusion CT is performed on an 8-cm segment (table toggle mode) centered on the circle of Willis; $40 \mathrm{~mL}$ of iodinated contrast material (Isovue 370; Bracco, Princeton, New Jersey) followed by $40 \mathrm{~mL}$ of saline is administered, and a series of dynamic low-dose scans $(80 \mathrm{kV}, 150$ $\mathrm{mAs}$ ) is obtained during injection. Contrast material is injected at $6 \mathrm{~mL} / \mathrm{s}$, the same rate used for CTA.

When perfusion CT is performed, the CTP data are used to generate a time-attenuation curve by placing the region of interest over the ICA. The time-to-peak enhancement of the arterial time attenuation curve is determined by choosing the peak enhancement level and assessing the time after injection at this point (typically approximately 16 seconds). For the combination CT angiograms of the head and neck typically performed with CTP, 2 seconds is subtracted from this time (typically approximately 14 seconds).

When CTP is not performed, a time-attenuation curve is generated over a single cervical carotid level (typically C3-C4). After the injection of $15 \mathrm{~mL}$ of iodinated contrast material (Isovue 370) and a 15-mL saline chaser, a series of dynamic 1-per-second lowdose scans $(80 \mathrm{kV}, 100 \mathrm{mAs})$ is obtained. Contrast material is injected at $6 \mathrm{~mL} / \mathrm{s}$. A region of interest is then placed over the ICA to generate the time-attenuation curve (Fig 1). The time-to-peak enhancement of the arterial time-attenuation curve is determined by choosing the peak enhancement level and assessing the time after injection at this point (typically approximately 14 seconds). For combination CT angiograms of the head and neck, this time is used as the scanning delay. For CT angiograms of the head alone (circle of Willis), 2 seconds is added to the time (yielding a total scanning delay of 16 seconds after initiation of contrast injection,

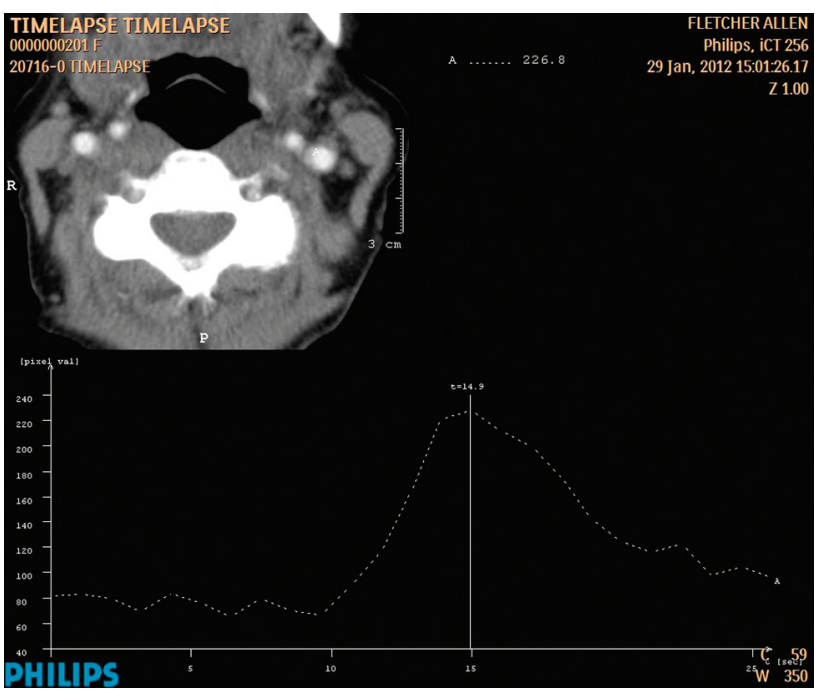

FIG 1. Time-attenuation curve obtained during preparation for 256detector CT angiography of the cerebrovasculature. The time attenuation curve reveals peak contrast at approximately 15 seconds (typically 14 seconds). For CTA of the neck (or CTA of the head and neck), this is the timing used as the scanning delay from the start of contrast injection. For CTA of the head only, 2 seconds is added (17 seconds in this example) to the delay time.

for example). Once the appropriate scanning delay has been determined, CTA is performed with $50 \mathrm{~mL}$ of iodinated contrast material (Isovue $370 \mathrm{I} / \mathrm{mL}$ ) followed by a $50-\mathrm{mL}$ saline bolus chaser at $6 \mathrm{~mL} / \mathrm{s}$ injection rate. Scanning parameters for CTA are as follows: number of detectors, 256; gantry rotation time, 0.5 seconds; caudocranial acquisition; collimation, $0.625 \mathrm{~mm}$; 0.985 pitch; $120 \mathrm{kV} ; 300 \mathrm{~mA}$. The 64-detector protocol uses the same gantry rotation time, collimation, pitch, $\mathrm{kV}$, and $\mathrm{mA}$, and the only difference is the number of detectors (ie, 64). Acquired data are then reconstructed at $0.9-\mathrm{mm}$ thickness with a $0.45-\mathrm{mm}$ reconstruction interval for evaluation of the vasculature, whereas the $0.625-\mathrm{mm}$ raw data images are used to create MIP images.

For contrast material infusion, an 18-gauge intravenous catheter is typically used. The right arm is preferentially selected to avoid streak artifacts from contrast material retained in the crossing left subclavian/brachiocephalic vein with a left-arm injection. These artifacts are more frequently encountered in elderly patients when the brachiocephalic vein is compressed between the sternum and an ectatic aortic arch. The intravenous catheter site is the antecubital fossa, over the forearm, with hand sites being chosen last. Delayed imaging of the head and neck is not routinely performed. A technologist performs image postprocessing at the scanner or in the 3D laboratory. MIP images are obtained of the level of the circle of Willis in axial, coronal, and sagittal planes; of the carotid bifurcations in sagittal oblique planes; and of the origins of the great vessels and extracranial carotid arteries with $360^{\circ}$ rotational images. Analysis of the degree of carotid artery stenosis is undertaken with the use of the North American Symptomatic Carotid Endarterectomy Trial criteria, in accordance with Medicare reimbursement. ${ }^{12}$

\section{Patient Population}

This retrospective study was conducted in accordance with the local Committee on Human Research in the Medical Sciences. 
Fifty consecutive patients presenting at our rural tertiary care referral center with concern for acute cerebrovascular attack who were evaluated with CTA and CTP on our 256-section emergency department CT scanner (Brilliance iCT; Philips, Best, the Netherlands) were included in this evaluation. Evaluated studies were performed between January 23, 2010, and June 2, 2011 (23 men, 27 women; mean age, 64.6 years; age range, $24-100$ years).

A comparison group of 50 consecutive patients presenting with the same indication who were scanned on the 64-section CT scanner (Brilliance CT; Philips) was also examined. These studies were performed between January 19, 2009, and April 20, 2009 (27 men, 23 women; mean age, 67.6 years; age range, $34-97$ years). This was the final period of time in which the 64-section scanner was located in the emergency department before being replaced with the 256-section scanner.

\section{Evaluation of Arterial Opacification}

The degree of arterial opacification was evaluated both quantitatively and qualitatively. Two neuroradiologists with certificates of added qualification in neuroradiology (C.G.F. and H.N.B.) with 6 and 16 years of experience performed a consensus qualitative evaluation of the entire study group for the contrast opacification of the carotid arteries according to a 5 -point scale $(5=$ excellent, 3 = good, 1 = poor). Each reviewer also separately reviewed a subset of 20 random cases to establish intraobserver consistency. The image quality of the proximal common carotid (approximately the level of the thyroid cartilage), distal common carotid (approximately the level of the hyoid bone), proximal internal carotid (just distal to the bifurcation), and the distal internal carotid arteries (at the level of $\mathrm{C} 1$ ) were assessed by these neuroradiologists, who were blinded to the patient's prior interpretation and clinical outcome. The percentage of diagnostic segments was calculated by dividing the number of adequately opacified vessels (defined as scores of 3-5) by the total number of vessels assessed. Occluded vessels were not evaluated with qualitative or quantitative measures but were subsequently correlated with other vascular imaging studies after completion of qualitative measurements to assess for true occlusion versus pseudo-occlusion.

Quantitative evaluation was performed on a separate workstation (MacPro, Apple, Cupertino, California) with the use of the OsiriX DICOM viewer (OsiriX Imaging Software; http://www. osirix-viewer.com), with placement a circular region of interest within each vessel at the 9 levels of interest; measurements were made in the aorta and the 4 levels of each carotid described above (Fig 2). The mean attenuation and standard deviation were measured for each artery by placement of a circular region of interest within the contrast-enhanced lumen of each segment. The regions of interest were sized to fill most of the intravascular space without including the vessel wall. Regions of interest typically measured approximately $10 \mathrm{~mm}^{2}$. Areas of vascular calcification were avoided. Adequate arterial opacification is regarded as $>150$ HU. Adequate arterial-venous segment contrast is regarded as $>50 \mathrm{HU}$.

Attenuation values of the veins at 9 corresponding locations were also noted: measurements were made in the superior vena cava and IJV on each side adjacent to the arterial measurement. To obtain the attenuation value, a circular region of interest (of

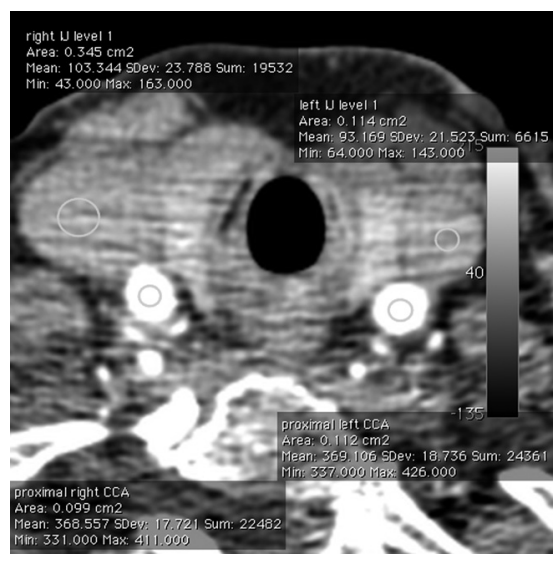

FIG 2. Axial image from a 256-detector CT angiogram at the level of the proximal CCA and proximal IJVs. Regions of interest show excellent arterial opacification (right CCA, $368 \mathrm{HU}$; left CCA, $369 \mathrm{HU}$ ) and sufficient contrast with the adjacent venous segment (right IJV, 103 $\mathrm{HU}$; left IJV, $93 \mathrm{HU}$ ). Mild venous enhancement is typical, given that CTP is performed before CT angiography.

the greatest diameter that would fit in the vessel lumen) was drawn on axial images in the center of the lumen. The typical size for such regions of interest was $15 \mathrm{~mm}^{2}$. The mean and SD values were recorded for each site. Both experienced CTA reviewers recorded artifacts during their evaluation of the carotid vasculature. Evaluation focused on artifacts that caused obscuration of arteries and limited diagnostic evaluation of that region. Artifacts were assigned to 1 of the 7 following categories:

1) Patient motion artifact.

2) "Streak" artifacts of venous origin (ie, attenuated contrast material in the subclavian vein, brachiocephalic vein, and/or superior vena cava).

3) "Streak" artifacts between the shoulders from photon starvation (low photon counts). This artifact becomes especially prominent at low doses and with thin-section imaging conditions.

4) Metallic streak artifact.

5) Contrast material reflux into small neck veins (ie, contrast in the vertebral venous plexus obscuring the adjacent vertebral artery).

6) Motion.

7) Flow artifacts or misrepresentation of flow dynamics (eg, pseudodissection or pseudo-occlusion). ${ }^{13}$

An examination was recorded as having an artifact if either reviewer recorded that it was present. Study timing was assessed as the complete examination time for the CTA of the head and neck, on the basis of information in the DICOM header. Dose-length products for the CTA (including the head and neck) were recorded from the dose report generated from the CT console and archived on the PACS.

\section{RESULTS}

Qualitative assessment of carotid opacification for the 256-section MDCT showed that of 391 arterial segments analyzed, 387 (99.0\%) of these segments had diagnostic opacification, as judged by both reviewers. The comparison evaluation of a historical group of patients evaluated on the 64-section MDCT revealed 


\begin{tabular}{|c|c|c|c|c|}
\hline & Description & $\begin{array}{l}\text { No. of Cases } \\
\text { (Incidence) on } \\
\text { 256-Section } \\
\text { CTA }\end{array}$ & $\begin{array}{l}\text { No. of Cases } \\
\text { (Incidence) on } \\
\text { 64-Section CTA }\end{array}$ & $\begin{array}{c}P \text { Value } \\
\text { (Fisher Exact } \\
\text { Test) }\end{array}$ \\
\hline Sufficient segments (qualitative) & $\begin{array}{l}\text { Segment graded as sufficient if graded as } \\
\text { adequate, good, or excellent }\end{array}$ & $387 / 391(99.0 \%)$ & $376 / 390(96.4 \%)$ & NS \\
\hline Sufficient segments (quantitative) & $\begin{array}{l}\text { Segment graded as sufficient if HU were } \\
>150\end{array}$ & $381 / 392(97.4 \%)$ & $365 / 390(93.6 \%)$ & NS \\
\hline $\begin{array}{l}\text { Sufficient segments (arteriovenous } \\
\text { contrast) }\end{array}$ & $\begin{array}{l}\text { Segment graded as sufficient if artery } \\
>50 \mathrm{HU} \text { above comparable venous } \\
\text { segment }\end{array}$ & $376 / 391(96.2 \%)$ & $361 / 390(92.6 \%)$ & NS \\
\hline
\end{tabular}

Note:-NS indicates not significant.

Table 2: Comparisons of artifacts limiting arterial evaluation with 256-section and 64-section CTA of the carotids

\begin{tabular}{|c|c|c|c|c|}
\hline Shoulder streak artifacts & $\begin{array}{l}\text { Photon starvation between shoulders obscures CCA, vertebral artery, IJV } \\
\text { in lower neck }\end{array}$ & 12 & 14 & 0.820 \\
\hline Metallic streak artifacts & $\begin{array}{l}\text { Dental hardware, cardiac defibrillator leads, spinal fusion hardware, etc, } \\
\text { obscure adjacent structures }\end{array}$ & 22 & 37 & 0.004 \\
\hline Contrast material reflux & Contrast reflux into veins of neck obscures adjacent vertebral artery & 0 & 0 & N/A \\
\hline $\begin{array}{l}\text { Flow artifacts } \\
\text { Arterial }\end{array}$ & $\begin{array}{l}\text { Altered flow dynamics (eg, slow flow) and rapid scanning time create } \\
\text { appearance of dissection flap or nonopacification of patent vessel }\end{array}$ & & & \\
\hline Pseudodissection & & 1 & 1 & 1.000 \\
\hline Pseudo-occlusion & & 0 & 1 & $\mathrm{~N} / \mathrm{A}$ \\
\hline Differential attenuation & & 5 & 1 & 0.204 \\
\hline Venous & & & & \\
\hline Pseudothrombosis & & 0 & 0 & N/A \\
\hline
\end{tabular}

that of 390 arterial segments analyzed, 376 (96.4\%) of these segments had diagnostic opacification. We did not find a statistically significant relationship between scanner type and the proportion of acceptable qualitative carotid segment opacification $(P=.91$, Fisher exact test). The qualitative evaluation of segments for each scanner also did not significantly differ $(P=1.00)$ with the quantitative evaluation (Table 1). Quantitative assessment of carotid opacification for the 256-section MDCT showed that of 391 arterial segments analyzed, 381 (97.4\%) of these segments had acceptable opacification and 376 of these segments (96.2\%) had acceptable contrast with the adjacent vein. The comparison evaluation of a group of patients evaluated on the 64-section MDCT revealed that of 390 arterial segments analyzed, 365 (93.6\%) of these segments had acceptable opacification and 361 of these segments (92.6\%) had acceptable contrast between the adjacent vein. We found no relationship between scanner type and the proportion of acceptable carotid segment opacification ( $P=1.0$, Fisher exact test). There was nearly complete concordance between the qualitative and quantitative assessment of the carotid segments through the use of the absolute attenuation measurements and the arterial contrast level within the adjacent vein.

The 10 cases (256-detector, $n=6$; 64-detector, $n=4$ ) with carotid segment nonfilling underwent further review with alternative modalities (Doppler sonography or conventional angiography) to assess for true occlusion versus pseudo-occlusion. All 10 cases were CTA after CTP. Six of the cases had sonography comparisons, which showed no detectable flow on Doppler. One case had conventional angiography, which revealed true occlusion, whereas 2 cases had no comparative imaging. One case (64-detector) of 8 with correlative imaging had a subsequent sonography, which revealed trace Doppler flow suggestive of CTA pseudoocclusion. Delayed CTA imaging was not part of our imaging protocol and was not performed in any of these cases.
An inter-rater reliability analysis with the use of the $\kappa$ statistic was performed to determine consistency between the 2 raters. The inter-rater reliability for the raters was $\kappa=0.90$. No rating differed by $>1$ point on the 5 -point scale.

The prevalence of different types of artifacts encountered on 256-detector CTA compared with 64-detector CTA is listed in Table 2. Only artifacts that caused obscuration of vessels were evaluated. No examination with the use of the 256-detector scanner or the 64-section scanner was associated with contrast material reflux into the IJV that limited arterial evaluation. On evaluation of the quantitative data, there was 1 examination for the 256-detector scanner and 2 for the 64-detector scanner in which there was significant quantitative reflux (as judged by proximal venous segment opacification greater than that of the adjacent arterial segment). This is in contrast to cases in which there is diffusely higher venous attenuation that is typically the result of delayed (or early) scanning phase (ie, "missed” bolus) (Fig 3). In each of the 3 cases of quantitatively identified internal jugular reflux, the degree of opacification in the adjacent arterial segments was adequate so that evaluation was not thought to be limited. There was no significant difference between 256- and 64-detector CTA in the incidence of artifacts except for those related to metallic streak artifacts, which were more often seen with 64-detector examinations ( $P=.004$, Fisher exact test). These were identified as limiting arterial evaluation in 37 segments (64detector examinations) compared with 22 segments (256-detector examinations). Flow-related artifacts occurred in 6 and 3 cases of 256- and 64-detector examinations, respectively, including the 1 case of 64-detector pseudo-occlusion described above. The 256section scan time was 4 seconds, compared with 6 seconds for the 64-section scan. The DLP for the 256-section CTA ( $M=1414$ mGy/ $\mathrm{cm}, \mathrm{SD}=113.9)$ was approximately $10 \%$ lower than the DLP of the 64-section CTA $(\mathrm{M}=1550 \mathrm{mGy} / \mathrm{cm}, \mathrm{SD}=159.8)(P<.001)$. 


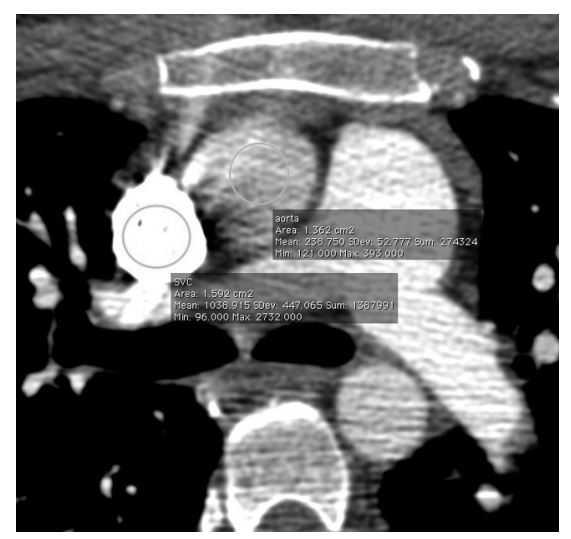

FIG 3. Axial image from a 256-detector CT angiograph at the level of the proximal ascending aorta and pulmonary artery. There is attenuated contrast within the pulmonary artery and superior vena cava $(1039 \mathrm{HU})$ compared with the lower attenuation of contrast within the proximal ascending aorta (239 HU). This pattern is typical for early scanning in which the contrast bolus has not yet maximally reached the arterial system.

\section{DISCUSSION}

CTA is generally accepted as the noninvasive criterion standard method for neurovascular evaluation in the setting of acute cerebrovascular attacks, on the basis of its speed, accuracy, and availability. ${ }^{14-17}$ Additionally, multimodality CT evaluation increases the sensitivity of stroke detection as well as in the prediction of the final infarct size over noncontrast CT alone. ${ }^{18-20}$ Evaluation of the 256-section CTA in our rural university-based tertiary referral hospital resulted in diagnostic quality studies in $>95 \%$ of cases, and the rate of appropriately opacified segments was not significantly different when compared with patients scanned at our institution with a 64-section CTA protocol, which is similar to the results achieved at other institutions in smaller published studies. ${ }^{13,21}$ Additionally, we further addressed the improvements in radiation dose to patients, which was typically $10 \%$ less with the 256-channel CTA study.

Proper timing of contrast material administration is a major determinant of optimal arterial opacification and CTA image quality. With scanners of 16- and 64-detectors, a fixed delay (usually approximately 25 seconds) between contrast injection and the start of scanning has been successfully used. ${ }^{22,23}$ However, given the physiologic variability of blood flow among patients (especially those with cardiac dysfunction) and the very fast imaging times of 256-section CT, accurately timing the delay between contrast material administration and scanning initiation with either a bolus-tracking technique or a preprocedure timing bolus becomes of greater importance than with previous generations of scanners. ${ }^{21,22,24}$ Unlike the work of Kim et al, ${ }^{13}$ who found the lowest mean opacification levels in the common carotid artery, we found equivalent opacification levels between the proximal common carotid artery and the distal internal carotid artery.

The 11 arterial segments in our study with attenuation $<150$ HU were approximately evenly distributed among the 4 vascular segments, which is also in contradistinction to prior work ${ }^{13,25}$ that described at least half of the poor quantitatively opacified segments to be in the proximal CCA. This may be in part due to the faster scanning times with the 256-detector scanner, our higher injection rate of $6 \mathrm{~mL} / \mathrm{s}$, and/or our slightly earlier scanning time of typically 14 seconds.

Although there is variability across centers in the order of performing CTP and CTA in emergent neurovascular evaluation, it has been shown that the order of the CTP and CTA has no significant influence on the quantitative or qualitative parameters of CTA intracranially or extracranially. ${ }^{26}$ Despite typical performance of CTP before performing CTA at our institution, the rate of acceptable contrast between the same arterial and venous segments ( $>50 \mathrm{HU}$ ) was $96.2 \%$ and $92.6 \%$ for the 256 -detector scanner and the 64-detector scanner, respectively. This rate of arterialvenous contrast was seen despite the presence of circulating background contrast from the CTP examination.

The predominant artifact identified in our study was related to metallic streak artifact typically caused by dental fillings and prostheses, and it was believed to degrade but not impede diagnostic image interpretation. Previously identified artifacts thought to be related to the "fast" scanning techniques seen with 64-detector and higher scanners, such as arterial pseudodissection, arterial pseudo-occlusion, and venous pseudothrombosis, were rarely seen in this study, and though there was a trend toward high flow-related artifacts in the 256-detector scans compared with 64-detector scans (6 examinations versus 3), this difference was not statistically significant $(P=.4870)$. Although these artifacts could be a source of potential confusion for those not familiar with "fast" scanning techniques, our experienced reviewers were able to differentiate true pathology from flow-related artifacts. A delayed CTA scan immediately after the arterial phase bolus administration is often necessary for full detection of critical stenoses to differentiate from a pseudo-occlusion and can also be useful in the setting of suspicious intracranial hemorrhage, but delayed scanning was not part of our imaging protocol. Other artifacts such as shoulder streak and contrast material reflux were not significantly different between the types of scanners.

Limitations of our study include the retrospective nature and bias in patient selection. Performing both 64-detector and 256detector CTA on the same group of patients would serve to reduce intersubject variation; however, logistical considerations prevented this. Only a small group of patients were identified who were evaluated on each scanner while similar scanning parameters were used. A relative limitation of our study was that we did not evaluate the accuracy of either the 256- or 64-section scanners compared with another technique (sonography, MR angiography, or digital subtraction angiography). However, the purpose of our study was not to evaluate the accuracy of a 256-section scanner but to assess the prevalence of adequate opacification of carotid segments.

An additional limitation of the fast scanning technique is the potential for overestimation of stenotic lesions ${ }^{27}$ and the identification of pseudo-occlusions. One case of CTA segmental nonopacification was confirmed with a follow-up sonography to be a patent segment, whereas 2 cases of CTA segmental nonfilling showed no detectable Doppler flow at follow-up sonography and 2 cases of CTA segmental nonfilling did not have correlative imaging. Although both interpreting neuroradiologists thought that these 2 cases with sonography follow-up did represent true occlusions, the lack of delayed CTA or DSA imaging prohibits defini- 
tive differentiation between a critical near-occluded segment and a completely occluded segment, given that the positive predictive value of sonography for carotid occlusion is only approximately 95\%. ${ }^{28-30}$ Future studies may benefit from additional correlation with other noninvasive imaging techniques or with subsequent imaging in selected patients.

\section{CONCLUSIONS}

With the use of similar techniques, 256-detector CTA evaluation of the extracranial carotid arteries provides adequate examination quality in approximately $95 \%$ of studies, similar to our experience with a 64-detector scanner. With the 256-detector CT scanner, there was a modest decrease in scan time, similar image quality, a reduction in radiation dose of approximately $10 \%$, and a reduction in the artifacts typically seen with the 64-detector CT.

\section{ACKNOWLEDGMENTS}

We wish to acknowledge George M. Ebert, $\mathrm{MD}, \mathrm{PhD}$, for his instrumental role in bringing this new technology to our center and teaching us how to use it.

\section{REFERENCES}

1. Murray CJ, Lopez AD. Mortality by cause for eight regions of the world: Global Burden of Disease Study. Lancet 1997;349:1269-76

2. Roger VL, Go AS, Lloyd-Jones DM, et al. Heart disease and stroke statistics-2011 update: a report from the American Heart Association. Circulation 2011;123:e18-e209

3. Gentile NT, Cernetich J, Kanamalla US, et al. Expedited computed tomography perfusion and angiography in acute ischemic stroke: a feasibility study. J Emerg Med 2011;43:308-15

4. Levitt MR, Ghodke BV, Cooke DL, et al. Endovascular procedures with CTA and MRA roadmapping. J Neuroimaging 2011;21:259-62

5. Schellinger PD, Fiebach JB, Hacke W. Imaging-based decision making in thrombolytic therapy for ischemic stroke: present status. Stroke 2003;34:575-83

6. Truwit CL. CT angiography versus MR angiography in the evaluation of acute neurovascular disease. Radiology 2007;245:362-66

7. Wardlaw JM, Stevenson MD, Chappell F, et al. Carotid artery imaging for secondary stroke prevention: both imaging modality and rapid access to imaging are important. Stroke 2009;40:3511-17

8. Johnson JM, Meader A, Hunt DT, et al. Pearls and pitfalls of noninvasive carotid imaging. In: Kalva S, Abbara S, eds. Vascular Imaging Pearls and Pitfalls. In press; 2012

9. Johnson JM, Lev ML. Computed tomography in acute stroke. In: Torbey M, Selim M. eds. The Stroke Brook. 2nd edition. In press; 2013

10. Hsiao EM, Rybicki FJ, Steigner M. CT coronary angiography: 256slice and 320-detector row scanners. Curr Cardiol Rep 2010;12:68-75

11. Mahesh M. Search for isotropic resolution in CT from conventional through multiple-row detector. Radiographics 2002;22:949-62
12. Seidenwurm D, Turski P, Barr J, et al. Performance measures in neuroradiology. AJNR Am J Neuroradiol 2007;28:1435-38

13. Kim JJ, Dillon WP, Glastonbury CM, et al. Sixty-four-section multidetector CT angiography of carotid arteries: a systematic analysis of image quality and artifacts. AJNR Am J Neuroradiol 2010;31:91-99

14. Lyden PD. Advanced brain imaging studies should not be performed in patients with suspected stroke presenting within $\mathbf{4 . 5}$ hours of symptom onset. Stroke 2011;42:2668-69

15. Parsons MW. Advanced brain imaging studies should be performed in patients with suspected stroke presenting within 4.5 hours of symptom onset. Stroke 2011;42:2666-67

16. Wintermark M, Albers GW, Alexandrov AV, et al. Acute stroke imaging research roadmap. Stroke 2008;39:1621-28

17. Yoo AJ, Pulli B, Gonzalez RG. Imaging-based treatment selection for intravenous and intra-arterial stroke therapies: a comprehensive review. Exp Rev Cardiovasc Ther 2011;9:857-76

18. Kloska SP, Nabavi DG, Gaus C, et al. Acute stroke assessment with CT: do we need multimodal evaluation? Radiology 2004;233:79-86

19. Young VEL, Sadat U, Gillard JH. Noninvasive carotid artery imaging with a focus on the vulnerable plaque. Neuroimaging Clin North Am 2011;21:391-405

20. Salottolo KM, Fanale CV, Leonard KA, et al. Multimodal imaging does not delay intravenous thrombolytic therapy in acute stroke. AJNR Am J Neuroradiol 2011;32:864-68

21. Siebert E, Bohner G, Dewey M, et al. 320-slice CT neuroimaging: initial clinical experience and image quality evaluation. Br J Radiol 2009;82:561-70

22. Bae KT. Test-bolus versus bolus-tracking techniques for CT angiographic timing. Radiology 2005;236:369-70

23. Delgado Almandoz JE, Romero JM, Pomerantz SR, et al. Computed tomography angiography of the carotid and cerebral circulation. Radiol Clin North Am 2010;48:265-81

24. Hallett RL, Fleischmann D. Tools of the trade for CTA: MDCT scanners and contrast medium injection protocols. Tech Vasc Interv Radiol 2006;9:134-42

25. de Monyé C, Cademartiri F, de Weert TT, et al. Sixteen-detector row CT angiography of carotid arteries: comparison of different volumes of contrast material with and without a bolus chaser. Radiology 2005;237:555-62

26. Dorn F, Liebig T, Muenzel D, et al. Order of CT stroke protocol (CTA before or after CTP): impact on image quality. Neuroradiology 2012;54:105-12

27. Jaff MR, Goldmakher GV, Lev MH, et al. Imaging of the carotid arteries: the role of duplex ultrasonography, magnetic resonance arteriography, and computerized tomographic arteriography. Vasc Med 2008;13:281-92

28. AbuRahma AF, Pollack JA, Robinson PA, et al. The reliability of color duplex ultrasound in diagnosing total carotid artery occlusion. Am J Surg 1997;174:185-87

29. Bekelis KN, Labropoulos N, Griffin M, et al, eds. Ultrasound and Carotid Bifurcation Atherosclerosis. London: Springer-Verlag; 2011

30. Mansour MA, Mattos MA, Hood DB, et al. Detection of total occlusion, string sign, and preocclusive stenosis of the internal carotid artery by color-flow duplex scanning. Am J Surg 1995;170:154-58 